\title{
Association of a Weakly Acidic Anti-Inflammatory Drug (Ibuprofen) with a Poly(Amidoamine) Dendrimer as Studied by Molecular Dynamics Simulations
}

\author{
I. Tanis and K. Karatasos* \\ Physical Chemistry Laboratory, Chemical Engineering Department, Aristotle University of Thessaloniki, \\ 54124 Thessaloniki, Greece
}

Received: April 28, 2009; Revised Manuscript Received: June 12, 2009

\begin{abstract}
In this work, we report results from fully atomistic molecular dynamics simulations regarding the associative behavior of a third-generation poly(amidoamine) dendrimer with ibuprofen, a weakly acidic nonsteroidal anti-inflammatory drug, in aqueous solutions and at different $\mathrm{pH}$ conditions. Employing a combined static and dynamic approach, we describe the specifics of the complexation/encapsulation of the drug within the dendritic structure. In addition, information regarding the dynamic behavior is provided for the self- and the collective motion of the drug molecules. The detail afforded by the present molecular-level description of the relevant associative mechanisms (i.e., electrostatic complexation, hydrogen-bonding), provides a deeper insight for the interpretation of recent experimental findings regarding the behavior of dendrimer/ibuprofen systems in an aqueous environment.
\end{abstract}

\section{Introduction}

Recent advances in pharmaceutical research have demonstrated that strategies based on a molecular-level design in drug formulations are superior as compared to conventional procedures (e.g., to systemic approaches ${ }^{1}$ ) in terms of their therapeutical efficiency. Key factors upon which the success of such formulations are based are the ability of the used systems to solubilize the desired drug compounds and to sustain an increased drug load while maintaining the integrity of the formed complexes under different thermodynamic and mechanical conditions as well as their ability to release the therapeutic agent in a controllable manner and to limit toxicity at minimum levels. ${ }^{2,3}$ A novel class of systems which to a large extent fulfills such requirements, combining at the same time advantageous features such as modifiable surface groups, multifunctionality, and nanoscale monodisperse size, are polymers bearing a regular dendritic architecture, referred to as dendrimers. ${ }^{3-5}$

These molecules can form covalent or noncovalent complexes with pharmaceutical compounds and act as vehicles for targeted drug delivery and controlled-release purposes. ${ }^{5,6}$ Complex formation with other compounds can be promoted, for example, by solvophobic/solvophilic interactions, hydrogen bonding, or ionic pairing or through chemical binding (conjugation) to their surface groups. ${ }^{7-9} \mathrm{~A}$ particular class of such molecules - namely, poly(amidoamine) dendrimers (PAMAM)-has been widely considered for biomedical applications. Properties such as biocompatibility, ${ }^{10}$ water solubility, versatility in modifying their functional groups, ${ }^{11}$ and responsiveness of their conformational properties to an aqueous environment ${ }^{12,13}$ render these molecules appropriate for such uses. These attributes enable PAMAM dendrimers to enhance drug solubilization and to facilitate the transport and the controlled release of complexed pharmaceutical and biological molecules at targeted sites. ${ }^{8,14,15}$

Among other biomedical uses, PAMAM dendrimers have recently emerged as promising candidates for the encapsulation and the delivery of poorly water-soluble drugs belonging to class

* To whom correspondence should be addressed. E-mail: karatas@ eng.auth.gr.
II compounds in the Biopharmaceutical Classification System ${ }^{16}$ (i.e., characterized by poor solubility but high permeability), which still pose a challenge in drug formulation. ${ }^{17-19}$ Although significant advances have been made through experimental observations regarding the association of such drug moieties with PAMAM dendrimers ${ }^{15,20-22}$ and several mechanisms have been proposed to explain the observed behavior, ${ }^{14,21,23}$ a molecular-level description that can offer both qualitative and quantitative information is particularly desirable if optimized formulations for such drugs are to be pursued.

To this end, we have employed fully atomistic molecular dynamics (MD) simulations, to examine the specifics of the association of a PAMAM dendrimer in aqueous solutions with a nonsteroidal anti-inflammatory drug (NSAID) with analgesic action, ibuprofen [2-(4-isobutylphenyl)propionic acid], which is used in the treatment of conditions such as rheumatoid arthritis, osteoarthritis, and ankylosing spondylitis. ${ }^{24}$ Ibuprofen (Figure 1) is a class II drug belonging to a category of weakly acidic pharmaceutical molecules bearing carboxylic groups (commercial compounds such as ketoprofen, diflunisal, naproxen, and indomethacin belong to the same category). ${ }^{22}$ Recent experiments have demonstrated that complexation of ibuprofen with PAMAM dendrimers not only can promote the solubilization of the drug but may increase its cellular uptake as well. ${ }^{21,25,26}$ It therefore represents an interesting system with good prospects for pharmaceutical uses, and a better understanding of the mechanisms involved in the complexation may offer valuable information toward setting a more general framework in the study of PAMAM/hydrophobic acidic drug systems.

Although ibuprofen can be administered as a racemic mixture of the two optical enantiomers ${ }^{27}$ (namely, $S$ and $R$ ), it is known that only the S-enantiomer is therapeutically active, ${ }^{27,28}$ whereas in vitro studies have demonstrated that administration of only the $S$-isomer can be advantageous due to the reduced metabolic load of the body and the better tolerance of patients to the drug doses. ${ }^{29}$ For these reasons, we have focused solely on the behavior of the $S$-isomer. Therefore, from now on when we use the term "ibuprofen" we will refer to the $S$-enantiomer. 

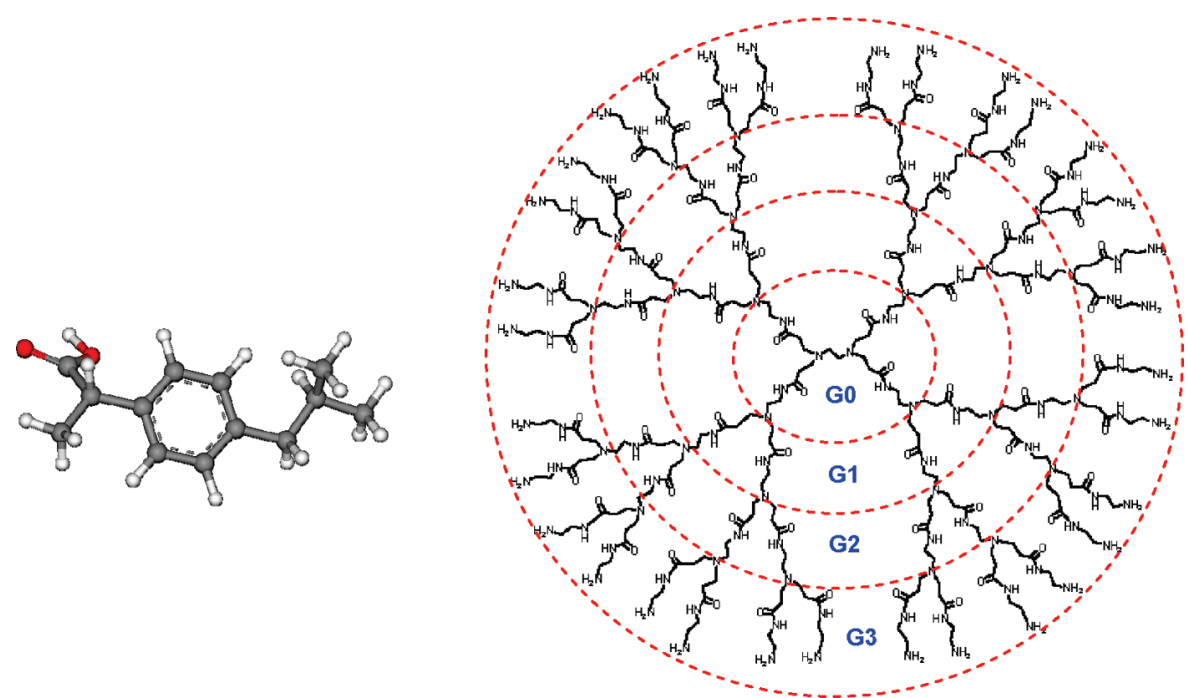

Figure 1. Left: The $(S)$-ibuprofen enantiomer (the carbon bonded to the carboxyl carbon is the chiral atom). Right: the third-generation $-\mathrm{NH}_{2}$ terminated poly(amidoamine) dendrimer examined in the present study. The dashed circles denote the boundaries between different generational shells.

\section{Simulation Details}

A. Description of the Models. We have considered ethyldiamine (EDA)-cored and - $\mathrm{NH}_{2}$-terminated PAMAM dendrimers of the third (G3) generation (Figure 1) in aqueous solutions (water molecules have been taken explicitly into account) in the presence of ibuprofen molecules. To mimic conditions representing different $\mathrm{pH}$ levels, we have simulated systems with the PAMAM dendrimer at various states of protonation of the amine groups. As follows from relevant titration experiments, ${ }^{30}$ at high $\mathrm{pH}$ conditions, PAMAM dendrimers are not charged; at physiological $\mathrm{pH}$, all primary amines (located at the periphery) are protonated; and at low $\mathrm{pH}$ values (i.e., $\mathrm{pH}<4$ ) the tertiary amines (located at the interior of the dendritic structure) become protonated, as well. Concerning the charging state of ibuprofen at different $\mathrm{pH}$ conditions, it has been found that at high $\mathrm{pH}$ conditions $(\mathrm{pH}>10.5)$, ibuprofen becomes fully ionized (due to deprotonation of the $-\mathrm{OH}$ of the carboxyl group), whereas at low $\mathrm{pH}$ conditions $(\mathrm{pH}<2)$, it remains neutral. ${ }^{14,21}$ Moreover, it was observed that the maximum load of ibuprofen molecules per dendrimer was realized when the drug was at its ionized state; that is, at a high $\mathrm{pH}$ environment. ${ }^{14,21}$ Specifically, it was demonstrated ${ }^{21}$ that for systems consisting of G3 PAMAM dendrimers, the maximum number of associated drug molecules per dendrimer did not exceed that of the primary amine groups of the dendrimer; that is, 32, regardless of the amount that was initially included in the solution. Taking into account the above experimentally available information, we have constructed models consisting of a G3 PAMAM dendrimer in different protonation states, water, and 32 ibuprofen molecules, as described in Table 1. To preserve the overall charge neutrality, an appropriate number of negative $\mathrm{Cl}^{-}$(in neutral and low $\mathrm{pH}$ conditions) and positive $\mathrm{Na}^{+}$(in the case of ionized ibuprofen) counterions was also added. ${ }^{31,32}$

As can be seen in Table 1, we have also considered a system consisting of a nonprotonated PAMAM dendrimer and unionized ibuprofen molecules (quoted as "G3_Basic") as a virtual reference state for comparison to the G3_Ionized system.

B. Construction and Simulation Protocol. Atomistic molecular models of the G3 PAMAM dendrimer were obtained from an earlier work. ${ }^{33}$ At the initial stage of the construction of the dendrimer/drug models, ibuprofen molecules were placed
TABLE 1: Details of the Simulated Systems ${ }^{a}$

\begin{tabular}{lccc}
\hline $\begin{array}{c}\text { systems" } \\
\text { notation }\end{array}$ & $\begin{array}{c}\text { total no. of } \\
\text { atoms in G3 } \\
\text { PAMAM dendrimer }\end{array}$ & $\begin{array}{c}\text { dendrimer } \\
\text { charge }\end{array}$ & $\begin{array}{c}\text { no. of solvent } \\
\text { molecules }\end{array}$ \\
\hline G3_Basic & 1092 & 0 & 4004 \\
G3_Neutral & 1124 & 32 & 6012 \\
G3_Acid & 1154 & 62 & 6033 \\
G3_Ionized & 1092 & 0 & 3189 \\
a The terms "Basic", "Neutral" and "Acid" refer to the pH \\
condition characterizing each system. The notation "ionized" refers \\
to the state of basic pH when all ibuprofen molecules are ionized.
\end{tabular}

within a spherical shell of width comparable to the dendrimer's radius of gyration around the dendrimer periphery (i.e., the maximum distance from the dendrimer's center of mass was twice the radius of gyration of the dendrimer). As noted earlier, to maintain charge neutrality, addition of an appropriate number of $\mathrm{Cl}^{-}$counterions in neutral and low $\mathrm{pH}$ conditions was made, whereas in the case of ionized ibuprofen, $\mathrm{Na}^{+}$counterions were included instead. The so-constructed dendrimer/drug models were solvated with explicit water molecules in a cubic simulation cell with dimensions sufficient to provide a solvation layer of at least $10 \AA$ around the dendrimer molecule ${ }^{34}$ (the size of the simulation box for all systems was several times larger compared to the radius of gyration of the dendrimer).

The systems were then subjected to energy minimization by at least 100000 steepest descent and conjugate gradient cycles by employing energetic parameters according to the $\mathrm{AMBER}^{35,36}$ forcefield for the dendrimer and ibuprofen molecules (including bond-stretching, angle-bending, torsional rotation, and van der Waals and hydrogen-bonding interactions) and the TIP3P set of parameters ${ }^{37}$ for the water molecules. Partial charges were assigned to the dendrimer/drug molecules by applying the Gasteiger algorithm, ${ }^{38}$ whereas electrostatic interactions were accounted for by full Ewald summation. van der Waals interactions were modeled by a 12-6 Lennard-Jones potential without taking into account interactions between atoms more than $10 \AA$ apart. Interactions between hydrogens of proton donors and proton acceptors were described by a $12-10$ potential term. ${ }^{39}$

Following the energy minimization, 200-600 ps MD simulations were performed in the constant-temperature, constant- 
pressure ensemble (NPT, $p=1 \mathrm{~atm}, T=300 \mathrm{~K}$ ), at the end of which the density of each system was stabilized. To further equilibrate the models in terms of dendrimer conformations and distributions of ibuprofen and water around the dendrimer, we performed a series of MD runs in the constant-temperature, constant-volume ensemble (maintaining the box size that was determined from the preceding NPT runs), starting from $800 \mathrm{~K}$ and cooling with steps of $100 \mathrm{~K}$ until a temperature of $300 \mathrm{~K}$ was reached. The systems spent at least 200 ps (in 1 fs steps) at each temperature. At the end of this procedure and at the $T$ $=300 \mathrm{~K}$ run, energy, pressure $(1 \mathrm{~atm})$, dendrimer size, and distributions of water and drug molecules around the dendrimer had reached equilibrium. Using these systems as starting points, we performed production runs of 4-6 ns in the microcanonical ensemble (NVE) with a time step of $1 \mathrm{fs}$ and frame-saving frequency of 1 ps. During production runs, all energetic components remained stable, the average pressure remained close to $1 \mathrm{~atm}$, and the temperature remained at $300 \mathrm{~K}$. The length of the produced trajectories was sufficient for the relaxation of the autocorrelation function describing fluctuations of the radius of gyration of the dendrimer molecules (not shown here).

To validate the adopted forcefield regarding the description of ibuprofen, we have also constructed a bulk model for the drug at $T=300 \mathrm{~K}$ following a similar combination of energy minimization and MD runs for the equilibration process. At the end of this cycle, the average density was stabilized to a value close to experimental, and energetic contributions had reached equilibrium, as well. Finally, trajectories of $4 \mathrm{~ns}$ length were performed in the NVE ensemble. During these runs, the drug sample remained at an amorphous state, as has been verified by checking the static structure factor arising from the centers of mass of the ibuprofen molecules (not shown here).

Utilization of the aforementioned forcefields (AMBER for the dendrimer and TIP3P for the water molecules) has been proven appropriate in the description of PAMAM dendrimers or other complex biological systems. ${ }^{40-43}$ To verify the suitability of the AMBER forcefield for the description of the ibuprofen molecules, we have checked characteristic properties such as the density, the Hansen solubility parameter ${ }^{44}$ and the average dipole moment of the drug and compared it to available literature data. Namely, after the NPT MD runs, the density was stabilized at $\rho=0.99 \pm 0.02 \mathrm{~g} / \mathrm{cm}^{3}$ which compares favorably to a value of $1.06 \mathrm{~g} / \mathrm{cm}^{3}$, as can be estimated for the amorphous state of ibuprofen, ${ }^{45,46}$ whereas calculation of the total Hansen solubility parameter $\delta$ based on the cohesive energy density (see, e.g., ref 47 for a more detailed description of the procedure) rendered a value of $9.57 \pm 0.46\left(\mathrm{cal} / \mathrm{cm}^{3}\right)^{0.5}$, which is within the range of 9.39 to $9.66\left(\mathrm{cal} / \mathrm{cm}^{3}\right)^{0.5}$ reported for bulk ibuprofen. ${ }^{48,49}$ Calculation of the average dipole moment of ibuprofen yielded a value of $1.80 \pm 0.02 \mathrm{D}$, which lies within the limits estimated for the more stable ibuprofen conformers. ${ }^{50}$

\section{Results and Discussion}

Previous studies on the solubility of poorly soluble acidic drugs in aqueous solutions of PAMAM dendrimers showed that drug/dendrimer complexes were formed, thus promoting the solubility of the hydrophobic drugs. ${ }^{3,51}$ These complexes were found to remain stable at neutral $\mathrm{pH}$ conditions, even after storage for several weeks, but they were found unstable in an acidic environment. Attempts for complex formation with nonpolar molecules were proven unsuccessful, ${ }^{51}$ suggesting that interactions between the polar regions of the acidic drug and the dendrimer were particularly important for complex forma- tion. On the other hand, the inability of the acidic drugs to be encapsulated within the dendrimer at low $\mathrm{pH}$ conditions where the tertriary amines lose their basic character indicated that an interaction between the acidic portion of the drug moiety and the basic tertiary amines of PAMAM molecules was likely to play a significant role in the complexation process. ${ }^{51}$ On the basis of further experimental studies in PAMAM/hydrophobic drug systems, it was proposed that such interactions between drug molecules and PAMAM amine groups could be of hydrogen bonding or electrostatic ${ }^{3,52}$ nature (or both). Particularly in the case of PAMAM/ibuprofen systems at high $\mathrm{pH}$ conditions where the drug is ionized while the dendrimer remains neutral, strong indications were found that complexation was realized through electrostatic interactions between the drug molecules and the peripheral amines of the dendrimer. ${ }^{14,21}$

From the aforementioned experimental findings, it follows that the mechanisms through which ibuprofen associates with PAMAM dendrimers as well as the longevity of the formed complexes correlate strongly to the charging state of the two kinds of molecules and the location of the ionized sites within the dendritic structure. To probe the level of association between ibuprofen and dendrimer and the location of the binding sites, we have examined characteristic static/structural properties of the systems as described in the following paragraph.

A. Spatial Arrangement of Ibuprofen Molecules and Complex Formation. Figure 2 depicts the density distributions of the dendrimer, the water, the ibuprofen molecules, and the counterions (if present), with respect to the center of mass of the dendrimer. As can be readily verified, in all cases, the water density reaches the bulk value at distances beyond the dendrimer's boundary (i.e., after the PAMAM density distribution drops to 0). In the basic $\mathrm{pH}$ regime (Figure 2a) (un-ionized ibuprofen), the dendrimer's distribution is characterized by a "dense core" conformation with a gradual drop toward the periphery. Water penetration is limited close to the dendrimer boundary, whereas ibuprofen penetration (although exhibiting a maximum close to the periphery as well) extents to the core region. In the neutral $\mathrm{pH}$ state (Figure $2 \mathrm{~b}$ ) where the primary amines of the dendrimer are protonated, the density distribution of the dendrimer (while still maximized close to the center-ofmass region) assumes lower values compared to the basic state and decays in a monotonic fashion toward the periphery, as has also been observed in earlier studies. ${ }^{31}$

Water molecules do not penetrate further than the dendrimer's boundary, as do the $\mathrm{Cl}^{-}$counterions, to neutralize the PAMAM protonated primary amines. It is interesting, though, to notice that the drug's profile appears more uniform compared to that characterizing the basic state (Figure 2a) and is shifted closer to the dendrimer's center of mass. In the low $\mathrm{pH}$ state (Figure $2 \mathrm{c}$ ), where both the primary and the tertiary PAMAM amines are protonated, the dendrimer distribution indicates again a dense core region, followed, however, by a marked drop in density. At distances further from the core, an almost constant density profile is formed, followed by the final drop near the periphery.

This behavior is consistent with the significant changes in the dendrimer's conformation at low $\mathrm{pH}$ conditions toward a more "open" structure, as has been observed in recent studies. ${ }^{13,31}$ Due to the increased availability of space in the dendrimer's interior, water molecules can penetrate even at distances near the center of mass, assuming an almost constant profile characterized by a value close to the density of bulk water.

The counterions' distribution changes accordingly, since now protonated amines can be found throughout the dendritic structure. Although the availability of free space within the 

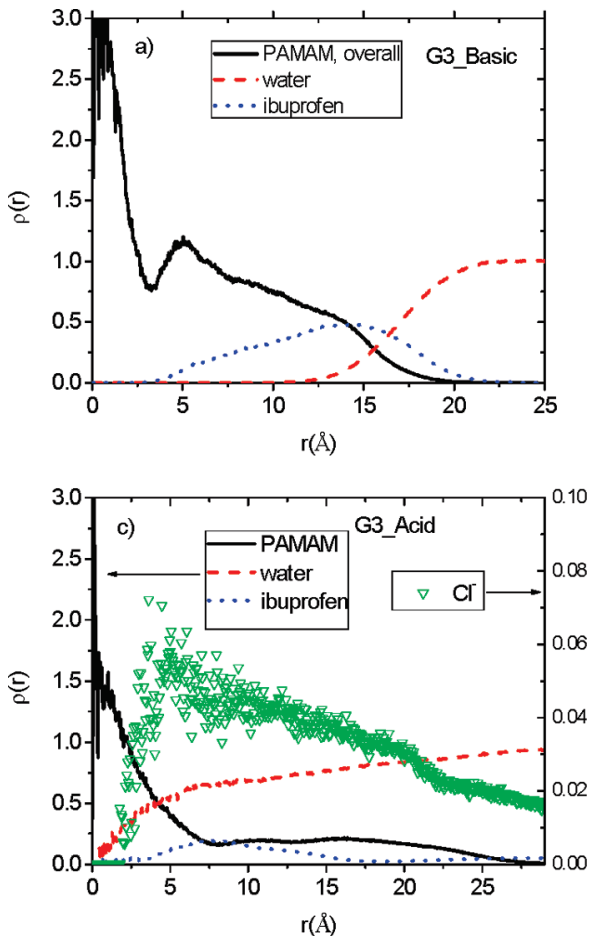
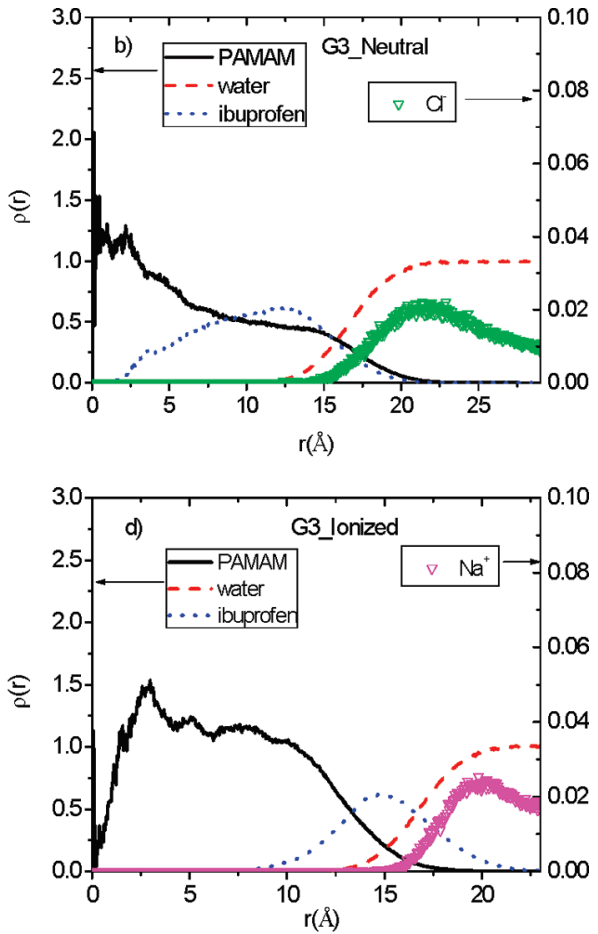

Figure 2. Density distributions of dendrimer, water, ibuprofen molecules, and counterions (if present) with respect to the center of mass of the dendrimer for all the examined systems (the notation of the systems follows that of Table 1).

dendrimer's interior becomes higher, the ibuprofen profile has dropped dramatically, indicating that whatever mechanism was responsible for the association of drug molecules with the dendrimer in the basic (Figure 2a) and neutral (Figure 2b) $\mathrm{pH}$ states becomes inactive at acid $\mathrm{pH}$ conditions.

In the high $\mathrm{pH}$ state where the carboxyl group of ibuprofen is negatively charged while the PAMAM amine groups are uncharged (Figure 2d), characteristic changes in both the dendrimer's and the drug's distributions take place. In comparison to the basic state (Figure 2a) where both molecular species are un-ionized, the dendrimer's profile does not exhibit a "dense core" behavior. Instead, a rather low-populated center of mass is followed by an almost homogeneous density distribution until the monotonic drop close to the periphery. Moreover, ibuprofen molecules are nearly normally distributed close to the outer PAMAM region. In view of the corresponding $\mathrm{Na}^{+}$density profile, it appears (note the overlap region between the drug and ion distributions) that a net charge due to the ionized drug is left close to the dendrimer's surface. The location of ibuprofen's density profile with respect to the dendrimer center of mass is a striking result, which clearly indicates a preferential physical binding of the drug molecules to the PAMAM's periphery. Although solely from this information no conclusion can still be drawn regarding the nature of the drug/dendrimer association, this finding is in qualitative agreement with relevant experimental studies, in which a linear dependence of ibuprofen's solubility was noted on the number of surface amines ${ }^{14}$ at high $\mathrm{pH}$ conditions. Furthermore, it is consistent with the observation that the number of ibuprofen molecules associated with a third-generation $-\mathrm{NH}_{2}$-terminated PAMAM dendrimer (identical to our model) in basic $\mathrm{pH}$ solutions was found to correspond to the number of the primary (outer) amine groups of the dendrimer. ${ }^{21} \mathrm{~A}$ visualization of the drug molecules' arrangement with respect to the dendrimer is presented in Figure 3. The arrangement of ibuprofen molecules relative to the dendrimer center of mass as described from the density distributions (Figure 2) can be recognized in the

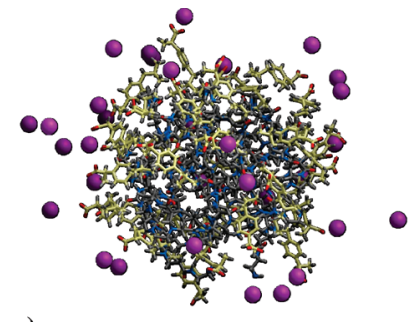

a)

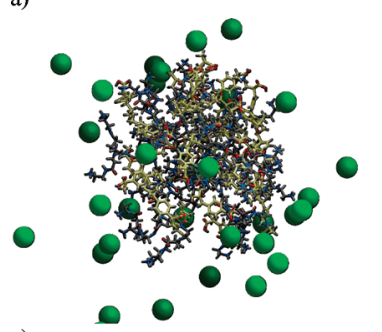

c)

b)

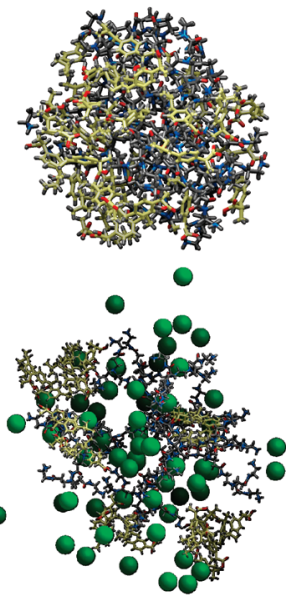

d)

Figure 3. Snapshots of the simulated systems: (a) basic $\mathrm{pH}$ with ionized drug (G3_Ionized), (b) basic pH (G3_Basic) without ionized species, (c) neutral pH (G3_Neutral), and (d) acid pH (G3_Acid) system. Water molecules are omitted for clarity. Ibuprofen molecules appear in lighter color for better visualization. Purple and green beads represent the $\mathrm{Na}^{+}$and $\mathrm{Cl}^{-}$counterions respectively.

snapshots. In the G3_Ionized system (Figure 3a), drug molecules are located only near the dendrimer's surface, forming a virtual shell around it. The picture for the G3_Basic system is similar to that for the G3_Ionized case, in which the drug molecules are, in principle, close to the dendrimer's periphery.

This is also consistent with the corresponding density profile (Figure 2a), where it was noted that the peak of the distribution was located close to the dendrimer's boundary. In the G3_Neutral system, all ibuprofen molecules appear to penetrate within the dendritic structure. In the G3_Acid system, although few drug molecules are located within the dendrimer's boundaries, it appears that ibuprofen molecules preferentially form clusters 


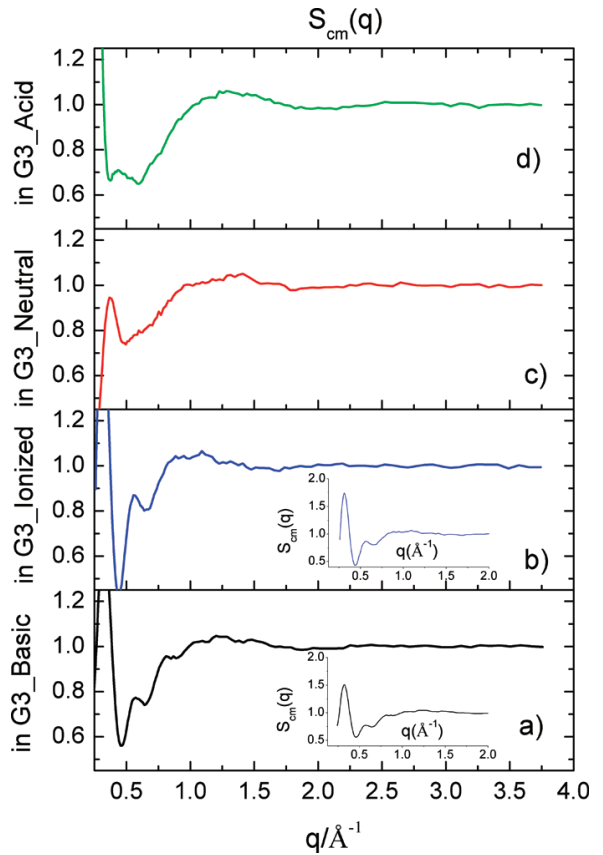

Figure 4. static structure factor arising from the centers of mass of ibuprofen molecules in the G3_Basic (a), the G3_Ionized (b), the G3_Neutral (c), and the G3_Acid (d) systems. Insets in parts a and b present a zoomed-out picture of the spectra in which the high amplitude peak at the low-q side is clearly discernible.

(we must also bear in mind the hydrophobic character of ibuprofen) instead of being homogenously dispersed within the dendrimer's interior.

To examine in more detail the relative placement of the ibuprofen molecules not only with respect to the dendrimer but also with respect to other ibuprofen molecules, we have calculated the static structure factor arising from the center of mass of the drug molecules (i.e., each drug molecule was considered as a single scatterer represented by its center of mass). To avoid any bias arising from an anisotropic arrangement of ibuprofen molecules in the solutions, we have calculated the structure factor by averaging over 20 directions uniformly distributed on the surface of a sphere for each magnitude of the scattering vector $|q|$ (henceforth denoted as $q$ ), as described in eq 1 .

$$
S_{\mathrm{cm}}(\mathbf{q})=\left\langle\left\langle\frac{1}{N}\left|\sum_{i=1}^{N} e^{i \vec{q} \cdot \vec{r}_{i}}\right| 2\right\rangle_{|\mathbf{q}|}\right\rangle_{\text {directions }}
$$

Figure 4 shows the so-calculated structure factor for all the examined systems. The features of the spectra in solutions at different $\mathrm{pH}$ levels exhibit characteristic differences. In the low pH state (Figure 4d), a low-amplitude maximum can be discerned at $q \cong 0.4 \AA^{-1}$, followed by a broad peak extending from $q \cong 1.0$ to $\cong 1.75 \AA^{-1}$. The former corresponds to a separation significantly larger compared to the size of an ibuprofen molecule. By the aid of the respective snapshot (Figure 3d) and taking into account the length scale corresponding to this $q$ value, we can assign this peak to the separation between the drug clusters. The broader peak at a larger $q$ value denotes a shorter characteristic distance, which can be ascribed to the separation between closest neighbors within the formed clusters (a peak approximately at the same location was also observed in the static structure factor of the bulk sample). This separation ranges between 3.5 and $6.2 \AA$, which lies between 1

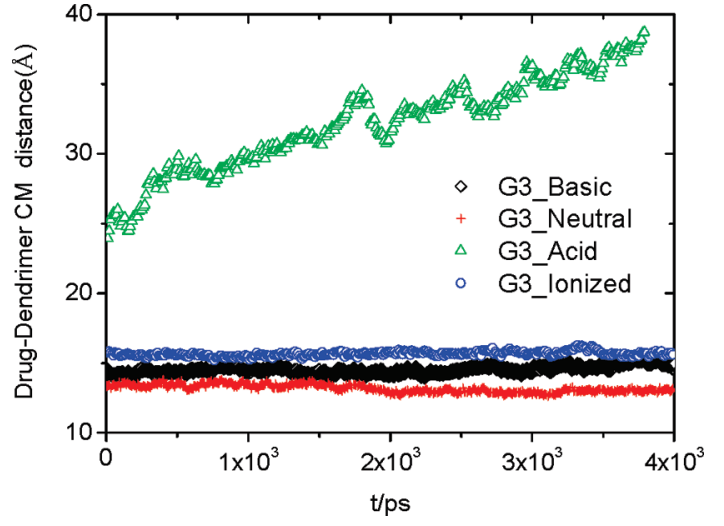

Figure 5. Average distance between the drug and the dendrimer centers of mass as a function of time within the examined window. The average has been performed over all ibuprofen molecules.

and 2 times the size of an ibuprofen molecule (the radius of gyration of a drug molecule is found to be $3.2 \AA$ ). The upturn at the low- $q$ side of the spectra is indicative of an even longerrange spatial correlation between ibuprofen molecules, which could be due to the fact that the relative distance between clusters may continue to grow as time lapses (see Figure 5 later in the text). Such a correlation length would have exceeded the size of the simulation box, and thus, a distinct peak could not have been observed in our analysis.

In the neutral $\mathrm{pH}$ state (Figure 4c), the broad peak observed at the low $\mathrm{pH}$ systems between 1 and $1.75 \AA^{-1}$ seems to be present, as well. A sharper maximum at $q \cong 0.38 \AA^{-1}$ represents a separation somewhat larger than the dendrimer's radius, which can be rationalized by the localization of the drug molecules within the dendritic structure (the maximum separation would correspond to twice the PAMAM radius). The basic $\mathrm{pH}$ systems exhibit certain features not observed in the low and neutral $\mathrm{pH}$ conditions: a low amplitude peak appears at $q \cong 0.56 \AA^{-1}$ together with a rather sharp maximum possessing much higher amplitude (see insets of Figure $4 \mathrm{a}$ and $\mathrm{b}$ ) at an even lower $q$ value of $q \cong 0.30 \AA^{-1}$. The high-amplitude, low- $q$ peak appears consistent with a separation very close to twice the radius of gyration of the dendrimer. Recalling the segregation of the drug molecules to the dendrimer's surface (Figures 2a, d and 3a, b), we can ascribe this peak to the distance between diametrically placed ibuprofen molecules around the dendrimer.

As far as it concerns the intermediate peak at $q \cong 0.56 \AA^{-1}$, we must assume that it arises from the manner that the drug molecules are arranged on the dendrimer's surface, since this peak can be observed only in systems bearing this specific geometry (i.e., G3_Basic and G3_Ionized). Actually, indications of higher-order peaks are present particularly in the G3_Ionized system, implying a well-defined spatial pattern in the drug molecule's arrangement close to the dendrimer's surface. A close examination of the snapshots in Figure $3 a$ and $b$ reveals the existence of "patches" of the dendrimer's surface not covered by drug molecules. Correlations between such drug-devoid areas on the surface or between local drug aggregations may result in the appearance of peaks possessing spectral features (i.e., relatively low amplitude due to the lower population and a $q$ magnitude corresponding to a separation that is shorter compared to twice the dendrimer's $\mathrm{Rg}$ ), similar to those characterizing the intermediate or higher-order peaks.

To examine the level of stability of the dendrimer/drug complexes, we have monitored the average distance between the ibuprofen and the PAMAM centers of mass as a function of time, as shown in Figure 5. Evidently, in basic and neutral 

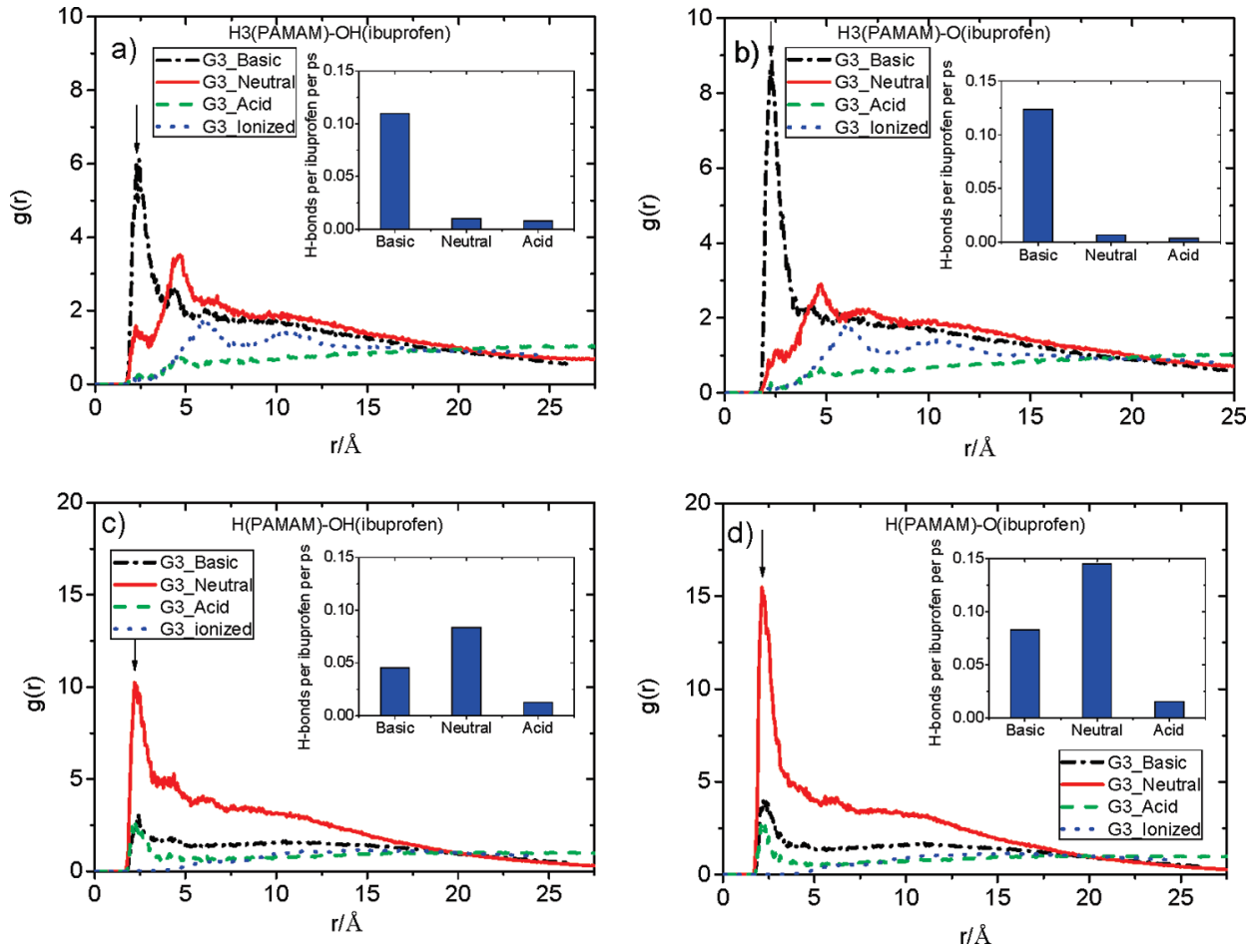

Figure 6. Radial distribution functions of hydrogen-bonding-capable pairs involving PAMAM hydrogen atoms and ibuprofen oxygens: (a) PAMAM amine hydrogen-ibuprofen hydroxyl oxygen, (b) PAMAM amine hydrogen-ibuprofen carbonyl oxygen, (c) PAMAM amide hydrogen-ibuprofen hydroxyl oxygen, (d) PAMAM amide hydrogen-ibuprofen carbonyl oxygen. Arrows indicate the location of the peak associated with hydrogen bonding. Insets present the number of hydrogen bonded pairs per ibuprofen molecule per time frame, averaged over all timeframes that are 1 ps apart. The relative error in the number of the hydrogen-bonded pairs does not exceed $10 \%$.

$\mathrm{pH}$ conditions, the drug molecules retain their average separation from the center of mass, indicating the formation of stable complexes (at least within our time window), in line with relevant experiments. ${ }^{21}$ In contrast, at acid $\mathrm{pH}$ conditions, they appear to diffuse away from the dendrimer, implying the eventual disruption of the dendrimer/drug contacts, again in qualitative agreement with pertinent experimental findings where no significant increase of the solubility of ibuprofen in the dendrimer solution was noted in a low $\mathrm{pH}$ environment. ${ }^{14}$

B. Nature of the Dendrimer/Drug Complexation. As has previously been noted, in dendrimer/drug systems ${ }^{8,53,54}$ and particularly in PAMAM complexes with acidic drug moieties (including ibuprofen), ${ }^{14,21,51}$ hydrogen bonding and ionic interactions are among those mechanisms suggested to play a decisive part in dendrimer/drug complexation. To determine the role of these mechanisms in PAMAM/ibuprofen association, we have examined radial distribution functions between characteristic atomic pairs which can potentially participate in such interactions. Namely, we examined characteristic hydrogen-acceptor pair distributions between PAMAM and ibuprofen atoms to detect the presence or absence of hydrogen bonding and thus to classify the complex formation as hydrogen-bonding- or non-hydrogen-bonding-driven. To this end, we checked PAMAM amine hydrogen (H3)-ibuprofen carbonyl oxygen (O), PAMAM amine hydrogen (H3)-ibuprofen hydroxyl oxygen (OH), PAMAM amide hydrogen $(\mathrm{H})$-ibuprofen carbonyl oxygen $(\mathrm{O})$, and PAMAM amide hydrogen $(\mathrm{H})$-ibuprofen hydroxyl oxygen $(\mathrm{O})$, representing PAMAM /drug hydrogen-bonding-capable atomic pairs.

The criteria we followed for identification of a hydrogen bond were based on the hydrogen-acceptor distance in conjunction with the angle formed by the donor-hydrogen-acceptor triplet. The maximum distance for counting the hydrogen-bonded pairs was determined by the extent of the corresponding peak in the pertinent pair distribution function, whereas only pairs for which the donor-hydrogen-acceptor angle was equal to or wider than $120^{\circ}$ were considered. ${ }^{41,55,56}$

Figure 6 shows the pair distribution functions between PAMAM amine/amide hydrogens and ibuprofen carbonyl/ hydroxyl oxygens. The peak indicative of hydrogen bond formation is marked by an arrow. Identification of hydrogenbonding maxima is based on the criteria mentioned earlier taking into account that $\mathrm{H} \cdots \mathrm{O}$ types of bonds as those examined here involve hydrogen-oxygen separations typically ${ }^{57,58}$ close to $2 \AA$.

From a visual inspection of the pair correlation functions, several general features can be noted. To begin with, in all the examined cases, hydrogen bond formation in low $\mathrm{pH}$ conditions appears either very rare or virtually absent. In addition, no indications for hydrogen bonding are observed in the G3_Ionized system. In contrast, in the G3_Basic system, hydrogen bonding between the drug and the dendrimer primary amines is rather frequent. It therefore follows that the mechanisms responsible for the preferential binding of the drug molecules close to the dendrimer's surface in the two cases at high $\mathrm{pH}$ conditions (Figures $3 a, b$ and $4 a, b$ ), are different. Participation of the amine hydrogens in hydrogen bonding in the neutral $\mathrm{pH}$ state is also quite infrequent. On the contrary, hydrogen bond formation at neutral $\mathrm{pH}$ conditions does take place between the amide hydrogen and ibuprofen molecules. This is also the case regarding the formation of hydrogen bonds between the dendrimer's carbonyl oxygens and the drug's hydroxyl hydrogens (not shown here). This finding appears consistent with the presence of drug molecules well within the interior of the dendritic structure at physiological $\mathrm{pH}$ conditions, as noted earlier (Figures $2 \mathrm{~b}$ and $3 \mathrm{c}$ ). A noticeable degree of hydrogen bonding is also observed between ibuprofen and PAMAM amide hydrogens in the G3_Basic system. Taking into account that amide hydrogens can be found at all generational shells (see 
Figure 1), the above observation is also compatible with the nonzero density profile of the drug within the dendrimer's interior (Figure 2a).

The picture emerging from the above behavior implies that at the neutral $\mathrm{pH}$ state physical association between drug molecules and dendrimer is to a large extent hydrogen-bondingdriven, due to hydrogen bond formation between PAMAM amide hydrogen (and PAMAM carbonyl oxygen sites, not shown here) and ibuprofen. Hydrogen bonding appears to play a significant role in the G3_basic system, as well, where ibuprofen molecules are associated mainly with the amine and, to a lesser extent, the amide hydrogens (and the carbonyl oxygens, not shown here) of the dendrimer. The rather rare hydrogen-bonding occurrence in the acidic state is consistent with the unstable character of PAMAM/drug complexes as described in Figure 5. On the other hand, the absence of any indication regarding dendrimer/drug hydrogen-bond formation in the experimentally realizable high $\mathrm{pH}$ state at which ibuprofen is ionized (G3_Ionized) indicates that a different mechanism should be responsible for the formation and the stability of these complexes. On the basis of the information obtained from the previous analysis, it seems plausible to exclude the possibility of hydrophobic interactions as being the principal mechanism acting in favor of complex formation at basic $\mathrm{pH}$ conditions; in such a case, ibuprofen would have rather been encapsulated in the dendrimer's interior instead of adopting an arrangement that far from minimizes the number of contacts with the aqueous environment. Therefore, bearing in mind the geometric characteristics of the drug molecules' arrangement around the dendrimer, their proximity to the outer amine groups (compare the pair distribution functions of the G3_Ionized system of Figure $6 a$ and $b$ with those of Figure $6 c$ and d) and the absence of hydrogen bonding, it appears reasonable to conclude that at high $\mathrm{pH}$ conditions where ibuprofen molecules are ionized, electrostatic pairing between the drug molecules and primary PAMAM amines can most likely account for the dendrimer/ drug complexation.

C. Dynamic Aspects of the Drug Behavior. Apart from the static picture concerning the arrangement of ibuprofen molecules in the formed complexes, it is of interest to examine the characteristic time scales describing self- and collective motion of the drug. This information could be important not only for the transport properties of the complexed drug but also for the optimization of the drug-target interactions when the complexes reach the targeted sites (e.g., cell membranes) and the drugrelease process. Self-motion of ibuprofen molecules was investigated by examining the incoherent dynamic structure factor arising from the centers of mass of the drug molecules according to eq 2

$$
S_{\text {inc }}(q, t)=\left\langle\frac{1}{N}\left\langle\sum_{i=1}^{N} \mathrm{e}^{-i \vec{q} \cdot\left[\vec{r}_{i}(t)-\vec{r}_{i}(0)\right]} \mid\right\rangle_{|q|}\right\rangle_{\text {directions }}
$$

To avoid effects associated with the system's anisotropy, for each scattering vector magnitude $q$, we have averaged over 20 different directions randomly distributed on a sphere's surface, as we did in the calculation of the static structure factor. In eq 2, $N$ represents the number of drug molecules (i.e., 32). This dynamic quantity essentially probes density fluctuations due to the movement of the individual monitored particles (here, the drug molecules), revealing both spatial and temporal characteristics of the motion. ${ }^{59}$

To obtain an insight regarding ibuprofen's self-motion, we have evaluated $S_{\text {inc }}(q, t)$ at characteristic scattering vector

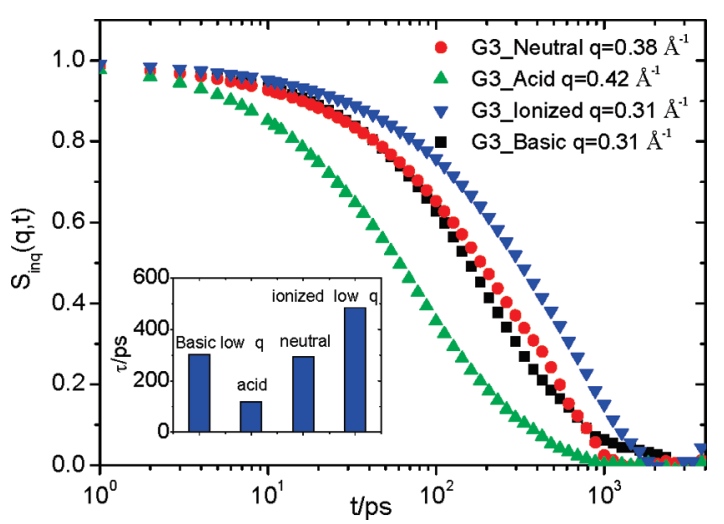

Figure 7. Incoherent dynamic structure factor of the examined systems at the lowest $q$ value corresponding to a peak of the static structure factor (Figure 4). Inset presents the characteristic relaxation times after integration of the respective spectra.

magnitudes; that is, corresponding to peak locations of the static structure factors (Figure 4).

Figure 7 illustrates the incoherent dynamic structure factors together with the average times estimated from integration of the corresponding spectra (for systems at basic $\mathrm{pH}$ conditions, spectra corresponding to the low-q peak are considered). The so-calculated times essentially provide a measure for the time scale corresponding to the slowest structural relaxation process of the drug molecules, at length scales not exceeding the overall size of the dendrimer.

Apart from the acid $\mathrm{pH}$ case, for the rest of the systems in which dendrimer/drug complexes appear to be stable, a time scale on the order of hundreds of picoseconds characterizes selfmotion of the drug molecules. Between the two systems in basic $\mathrm{pH}$ conditions, the longest time corresponds to the G3_Ionized system, indicating that the mechanism responsible for dendrimer/ drug complexation in this system (i.e., ionic pairing), induces a more constricted motion of ibuprofen compared to that in the hydrogen-bonding-sustained complex (G3_Basic system). The shorter time scale in the G3_Acid system is consistent with the absence of dendrimer/drug complexation and, thus, with a less restricted motion of drug molecules either in an individual or in a cluster state.

Figure 8 presents the $q$-dependence of the self-motion of ibuprofen molecules as expressed by $S_{\text {inc }}(q, t)$ in a wide $q$ range, covering larger as well as shorter length scales compared to the dendrimer dimensions. At the low- $q$ range (i.e., at length scales larger than the dendrimer dimensions), drug molecules exhibit a diffusive motion (i.e., $\tau^{-1} \propto q^{2}$ ) independently of their associative state with the dendrimer. For those systems in which drug molecules are complexed with the dendrimer (i.e., all but the G3_Acid system), this behavior essentially reflects the diffusive motion of the dendrimer/drug complex. For the G3_Acid system, it can be the result of the diffusive motion either of individual molecules or of the hydrophobically sustained drug clusters.

Small differences in the absolute values of the characteristic times should be attributed to the slower or faster diffusive motion of the different systems with respect to each other. For instance, the fact that the G3_Ionized system exhibits somewhat longer times implies a larger hydrodynamic radius of this system as compared to the rest. As the length scale approaches that of the dendrimer dimensions, a differentiation of the time scales for the individual drug motion takes place, depending on the system examined. The $q$ range at which this change is realized, denoted by the hatched area in Figure 8, coincides with the range 


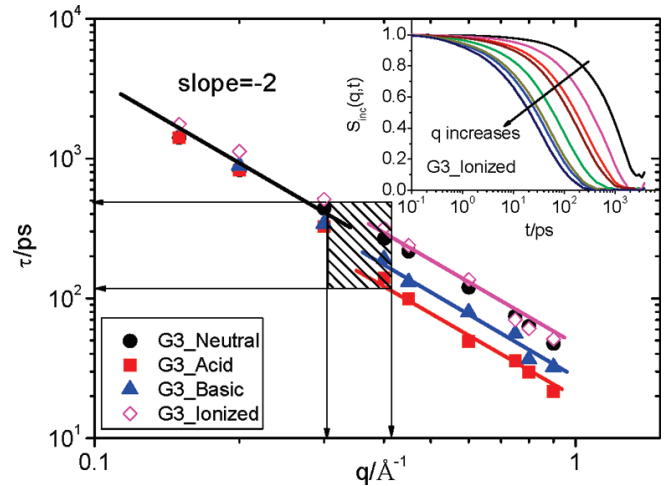

Figure 8. $q$ Dependence of the average times, $\tau$, of $S_{\text {inc }}(q, t)$ at $q$ values covering length scales below and above the dendrimer dimensions. The arrows mark the $q$ limits corresponding to the location of the lowest- $q$ peaks appearing to the static structure factor of the centers of mass (Figure 4) and the time limits associated with the respective $S_{\text {inc }}(q, t)$ spectra (Figure 7, inset). Lines denote a slope of -2 . Inset depicts an example of $S_{\text {inc }}(q, t)$ spectra at different $q$ values for one of the examined systems (the behavior of spectra describing the rest of the systems is analogous). The $q$ values of the spectra shown in ascending order are $0.2,0.30,0.4,0.45,0.6,0.75,0.80$, and $0.90 \AA^{-1}$.

at which the static structure factor peaks appear (Figure 4); that is, at length scales at which spatial correlations between drug molecules are established. The time scale separation observed between systems at higher $q$ values (i.e., at shorter distances) implies that distinct dynamic environments are experienced by the drug molecules in different systems, in line with the different degree of constriction imposed by their geometrical arrangement, as described earlier. In the higher $q$ limit examined, where the respective length scales become comparable to few drugmolecule radii (i.e., at $q>0.7 \AA^{-1}$ corresponding to separations shorter than $\sim 9 \AA$ ), deviations from the immediately lower $q$ regime behavior are observed in the complexed systems. Such deviations are most probably associated with the non-Gaussian character of the drug molecules' displacement at such short distances and to the more constricted environment. ${ }^{60,61}$

To explore the collective motion of ibuprofen, we have examined the distinct Van Hove correlation function (eq 3), which probes density fluctuations due to the cooperative motion of the neighbors around each particle ${ }^{59}$ (here a drug molecule).

$$
G_{\mathrm{d}}(\mathbf{r}, t)=\frac{1}{N}\left\langle\sum_{i} \sum_{j \neq i} \delta\left[\mathbf{r}-\left|\mathbf{r}_{i}(t)-\mathbf{r}_{j}(0)\right|\right]\right\rangle
$$

In the former expression, $N$ represents the number of particles (here, 32), while $i$ and $j$ refer to drug-molecule indices. This function is proportional to the probability that a particle is at position $\mathbf{r}$ at time $t$, provided that a different particle was at the origin $(\mathbf{r}=0)$ at time $t=0$. Its Fourier transform is the coherent dynamic structure factor which can be probed by experimental techniques possessing spatial and temporal resolution, such as neutron scattering. ${ }^{62}$ At $t=0$, the distinct Van Hove function is proportional to the radial distribution function $g(r)$ of the examined particles. At large times and long separations, the position of each particle is uncorrelated to the position of another particle at earlier times, which leads to the gradual smearing out of the $g(r)$ peaks. To facilitate a visual inspection of the rate at which the shell formed by the nearest neighbors loses cohesion, we have normalized the $y$-axis with respect to the amplitude of the first-neighbor peak corresponding to the static case (i.e., at $t=0$ ).
Figure 9 illustrates the behavior of $G_{\mathrm{d}}(r, t) / G_{\mathrm{d}, \max }(r, 0)$ of the examined systems covering a wide range of time scales. As time lapses, the peak indicating the spatial correlation with the nearest neighbors becomes broader and gradually loses its amplitude due to the disruption of the transient "cage" formed by the surrounding drug molecules. At the same time, at longer time scales and at distances close to $\mathbf{r}=0, G_{\mathrm{d}}(r, t)$ increases. This increase of the distinct Van Hove function at $\mathbf{r}$ close to 0 , signifies that particles (i.e., drug molecules) that were at $\mathbf{r}=0$ at $t=0$ are being substituted by other particles as time progresses. In other words, this behavior reflects a dynamic exchange between drug molecules.

Actually, we can identify a characteristic time $t_{\max }$ above which the level of $G_{\mathrm{d}}(r, t)$ does not increase anymore, or it starts decreasing. ${ }^{63,64}$ This behavior is illustrated in the insets of Figure 9. Both in main panels and in insets, arrows point to the direction of the increase of time. At the time scale corresponding to $t_{\max }$, the probability that a site which was originally occupied by a particle is at a later time occupied by a different particle is maximized. This time scale can be taken as a measure for the average time required in order that a particle is substituted in position with a different one; that is, a cooperative rearrangement between particles has been realized. Apparently (see insets), this time scale depends on the system under examination. For the G3_Basic system, $t_{\max }$ is estimated close to $400 \mathrm{ps}$; for the G3_Ionized, close to $800 \mathrm{ps}$; for the G3_Neutral, close to $1 \mathrm{~ns}$; and for the G3_Acid, close to 300 ps. Comparing the time scales for collective modes to those describing self-motion at length scales close to dendrimer dimensions (Figure 7 inset), it appears that the former are at all cases longer, as expected due to their cooperative nature.

This dynamic information implies a rather simple picture as far as it concerns the degree of difficulty in the realization of the collective spatial rearrangement of drug molecules, which is consistent with the static picture described earlier. Namely, it can be seen that it takes much more time (i.e., it is much more difficult) for drug molecules to rearrange themselves in a cooperative manner when located in the dendrimer's interior and having formed hydrogen-bonds with it (as is the case for the G3_Neutral system) than to move collectively when being close to the surface (as is in the basic pH state, G3_Basic and G3_Ionized systems) or not being associated with the dendrimer at all, as is the case in low $\mathrm{pH}$ conditions. Moreover, the fact that between the two systems at basic $\mathrm{pH}$ conditions a longer $t_{\max }$ corresponds to the G3_Ionized system implies that the physical binding to dendrimer sites is firmer in that case. Particularly in the two models at high $\mathrm{pH}$, a characteristic "jump" of the $G_{\mathrm{d}}(r, t)$ can be observed between 100 and 200 ps (pointed out by vertical arrows in Figure 9a and b), not detected in the other systems. Taking into account the arrangement of ibuprofen molecules near the dendrimer's surface (a characteristic that differentiates these systems from the others), we can surmise that at that time scale, a particular synergistic dynamic process associated with position exchange between neighboring drug molecules takes place.

\section{Summary/Conclusions}

Examination of static and dynamic properties of dendrimer/ ibuprofen systems revealed that distinct mechanisms are responsible for the formation of dendrimer/drug complexes at different $\mathrm{pH}$ conditions in aqueous solutions. At low $\mathrm{pH}$, no stable dendrimer/drug complex was detected (see Figure 5), in agreement with relevant experimental observations. ${ }^{14}$ Despite the better accessibility of drug molecules to PAMAM hydrogen- 

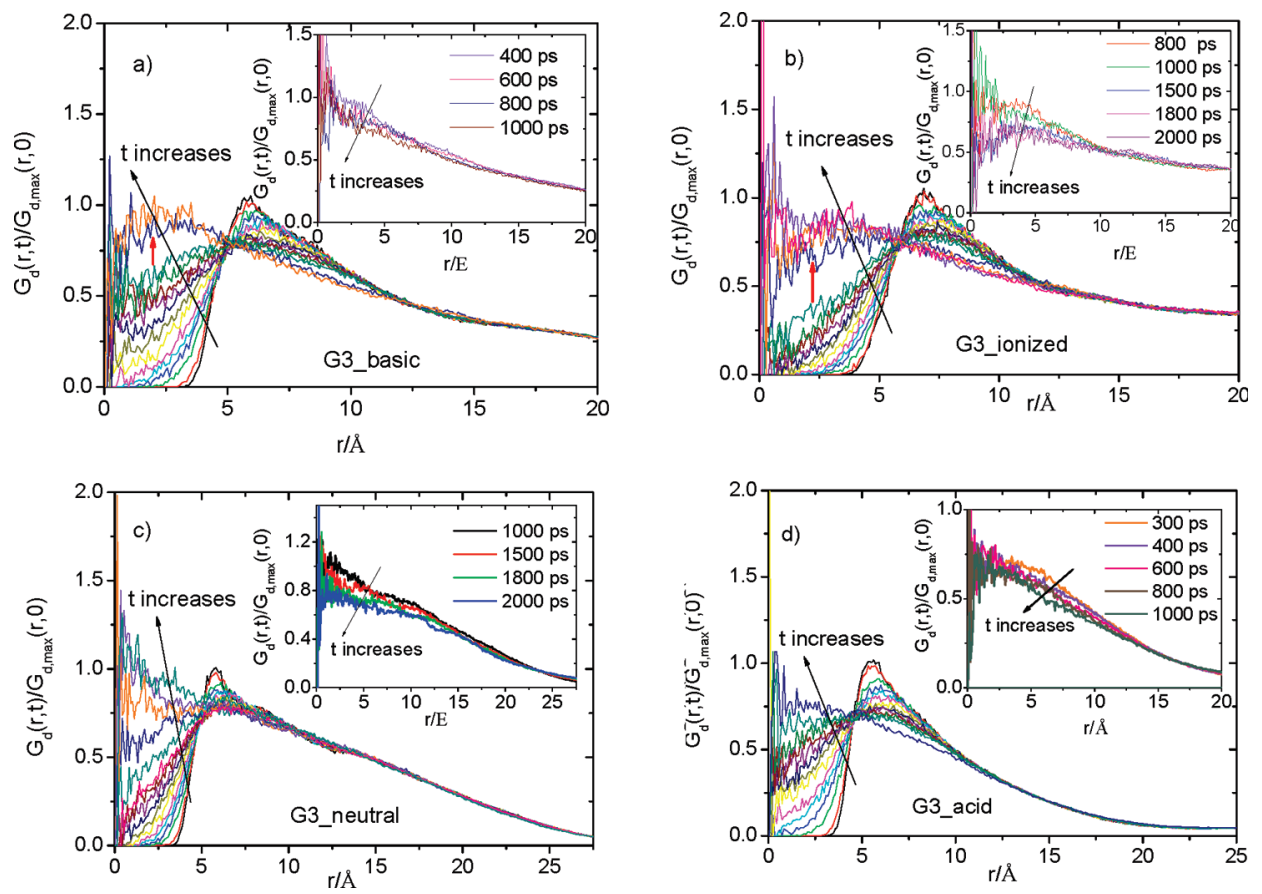

Figure 9. Distinct Van Hove space-time correlation functions arising from the centers of mass of the drug molecules for all the examined systems, normalized to the value of the maximum at $t=0$. The sequence of times (in ps) corresponding to the curves shown is $0,1,5,10,15,20,30,40$, $50,60,70,80,100,200,300,400,600,800,1000,1500,1800$, and 2000. Insets show the behavior of the district van Hove function, from the time scale beyond which a decrease in the low-r region is observed.

bond-capable sites due to the more "open" conformation adopted by the dendrimer, hydrogen-bonding between dendrimer and drug molecules was practically absent. In such conditions, instead of associating with the dendrimer (an occurrence that would have increased the drug's solubility), the hydrophobic drug molecules form clusters, which eventually diffuse away from it, thus keeping the solubility of the drug at levels similar to those expected in the absence of dendrimer.

At physiological $\mathrm{pH}$ conditions, dendrimer/drug complexation is promoted mainly via hydrogen bonding between ibuprofen molecules and PAMAM amide hydrogens and carbonyl oxygens, leading to the formation of stable complexes. In this system, drug molecules appear to penetrate well within the dendritic structure, which undergoes a conformational change upon protonation of the primary amines. The protonated amine groups do not appear to participate in hydrogen bond formation.

In the basic $\mathrm{pH}$ state in which ibuprofen molecules are ionized, stable PAMAM/ibuprofen complexes are formed, as well; however, neither hydrogen bonding nor hydrophobic interactions appear to act as driving forces for complexation. On the basis of this information and the fact that ibuprofen molecules are found to preferentially arrange themselves at the dendrimer's surface, it is concluded that electrostatic interactions between the drug and the primary amine groups is the principal reason for the observed behavior. This conclusion is in a good agreement with the mechanism suggested from relevant experimental studies. ${ }^{14,21}$ An analogous associative behavior is exhibited by the virtual system in which neither the dendrimer nor the drug molecules are ionized; however, complex formation in that case appears to be hydrogen-bonding-driven, mainly due to the formation of hydrogen bonds between ibuprofen and the primary dendrimer amines. In other words, ionization of ibuprofen clearly makes the difference in the associative behavior between the dendrimer and the drug at high $\mathrm{pH}$ conditions.

The dynamic properties of ibuprofen molecules are found to be correlated to the specifics of their spatial arrangement with respect to the dendrimer. At low $\mathrm{pH}$ conditions, both self- and collective drug dynamics appear to be faster as compared to the systems at higher $\mathrm{pH}$ levels and to lie close to $100 \mathrm{ps}$ (taking as scattering vector the one corresponding to the low- $q$ peak of the static structure factor; Figure 4a) and 300 ps, respectively. In the neutral $\mathrm{pH}$ state, the dynamic behavior of the formed dendrimer/drug complex indicates slower-moving drug molecules characterized by a sluggish collective motion, compatible with the spatially constricted arrangement of the molecules revealed from the static picture. The time scale for self-motion at separations within the dendritic structure is of the order of several hundred ps $(\sim 300$ ps at the low- $q$ peak of the static structure factor; Figure $4 \mathrm{c}$ ), whereas the average time scale for collective rearrangements reaches the nanosecond range.

At high $\mathrm{pH}$ conditions, self- as well as collective dynamics in the ionized ibuprofen system appears slower as compared to that characterizing the system with nonionized ibuprofen molecules. This observation implies a stronger physical binding between dendrimer and drug when the latter is ionized, consistent with the electrostatic nature of the dendrimer/drug interaction. In addition, the G3_Ionized system assumes the slower self-motion from all the examined systems ( $\sim 500 \mathrm{ps})$, wehreas the time scale for collective motion is comparable (but somewhat faster) compared to that characterizing the neutral $\mathrm{pH}$ state, despite the absence of an apparent geometrical confinement in a sense similar to that observed in the latter. Due to the arrangement of drug molecules at the surface of the dendrimer at high $\mathrm{pH}$ conditions, it appears that specific dynamic mechanisms may facilitate close-neighbor position exchanges, thus accelerating collective drug motion.

Although the above-described static and dynamic picture refers to complexation of PAMAM dendrimers with a specific drug, ibuprofen, we believe that the mechanisms responsible for the dendrimer/drug complexation described here may share to a large extent common characteristics with those acting on systems of PAMAM dendrimers with a broader category of carboxyl-bearing weakly acidic pharmaceutical compounds. 
Acknowledgment. Funding from the Greek General Secretariat for Research and Technology and the European Community under the framework of the PENED 2003 program (Grant 03E 4716 ) is gratefully acknowledged. Part of the computing time for this work was provided through a CPU grant (project SH-024-07) from The Netherlands Organization for Scientific Research (National Computing Facilities Foundation).

\section{References and Notes} 311.

(1) Grainger, D. W.; Okano, T. Adv. Drug Delivery Rev. 2003, 55,

(2) Torchilin, V. Multifunctional Pharmaceutical Nanocarriers: Development of the Concept. In Fundamental Biomedical Technologies, Multifunctional Pharmaceutical Nanocarriers; Springer: New York, 2008.

(3) Beezer, A. E.; King, A. S. H.; Martin, I. K.; Mitchel, J. C.; Twyman,

L. J.; Wain, C. F. Tetrahedron 2003, 59, 3873.

(4) Svenson, S. Eur. J. Pharm. Biopharm. 2009, 71, 445.

(5) Palsson, L. O.; Tsiourvas, D.; Sideratou, Z.; Tziveleka, L. A. Curr. Top. Med. Chem. 2008, 8, 1204.

(6) Navath, R. S.; Kurtoglu, Y. E.; Wang, B.; Kannan, S.; Romero, R.; Kannan, R. M. Bioconjugate Chem. 2008, 19, 2446.

(7) Wang, C.; Wyn-Jones, E.; Sidhu, J.; Tam, K. C. Langmuir 2007, 23,1635 .

(8) Fernandez, L.; Gonzalez, M.; Cerecetto, H.; Santo, M.; Silber, J. J. Supramol. Chem. 2006, 18, 633.

(9) Devarakonda, B.; Hill, R. A.; de Villiers, M. M. Int. J. Pharm. 2004, 284, 133.

(10) Malik, N.; Wiwattanapatapee, R.; Klopsch, R.; Lorenz, K.; Frey, H.; Weener, J. W.; Meijer, E. W.; Paulus, W.; Duncan, R. J. Controlled Release 2000, 65, 133.

(11) Perumal, O. P.; Inapagolla, R.; Kannan, S.; Kannan, R. M. Biomaterials 2008, 29, 3469.

(12) Chen, W.; Tomalia, D. A.; Thomas, J. L. Macromolecules 2000 33,9169 .

(13) Lee, I.; Athey, B. D.; Wetzel, A. W.; Meixner, W.; Baker, J. R. Macromolecules 2002, 35, 4510.

(14) Milhem, O. M.; Myles, C.; McKeown, N. B.; Attwood, D.; D'Emanuele, A. Int. J. Pharm. 2000, 197, 239.

(15) Cheng, Y.; Xu, T. Eur. J. Med. Chem. 2005, 40, 1188.

(16) Amidon, G. L.; Lennernas, H.; Shah, V. P.; Crison, J. R. Pharm. Res. $1995,12,413$.

(17) Rawlinson, C. F.; Williams, A. C.; Timmins, P.; Grimsey, I. Int. J. Pharm. 2007, 336, 42.

(18) Garzón, L. C.; Martínez, F. J. Solution Chem. 2004, 33, 1379.

(19) Chan, K. L. A.; Kazarian, S. G. J. Comb. Chem. 2006, 8, 26.

(20) Cheng, Y.; Xu, T.; Fu, R. Eur. J. Med. Chem. 2005, 40, 1390.

(21) Kolhe, P.; Misra, E.; Kannan, R. M.; Kannan, S.; Lieh-Lai, M. Int. J. Pharm. 2003, 259, 143.

(22) Cheng, Y.; Man, N.; Xu, T.; Fu, R.; Wang, Xueyuan; Wang, Xiaomin; Wen, L. J. Pharm. Sci. 2007, 96, 595.

(23) D'Emanuele, A.; Attwood, D. Adv. Drug Delivery Rev. 2005, 57, 2147.

(24) Park, E. S.; Chang, S. Y.; Hahn, M. Y.; Chi, S. C. Int. J. Pharm. 2000, 209, 109.

(25) Kannan, S.; Kolhe, P.; Raykova, V.; Glibatec, M.; Kannan, R. M.; Lieh-Lai, M.; Bassett, D. J. Biomater. Sci., Polym. Ed. 2004, 15, 311.

(26) Kolhe, P.; Khandare, J.; Pillai, O.; Kannan, S.; Lieh-Lal, M.; Kannan, R. M. Biomaterials 2006, 27, 660.

(27) Williams, K.; Day, R.; Knihinicki, R.; Duffield, A. Biochem. Pharmacol. 1986, 35, 3403. 418
(29) Stock, K. P.; Geisslinger, G.; Loew, D.; Beck, W. S.; Bach, G. L.; Brune, K. Rheumatol. Int. 1991, 11, 199.

(30) van Duijvenbode, R. C.; Borkovec, M.; Koper, G. J. M. Polymer 1998, 39, 2657.

(31) Maiti, P. K.; Çağin, T.; Lin, S. T.; Goddard, W. A. Macromolecules 2005, 38, 979 .

(32) Jusufi, A.; Hynninen, A.-P.; Panagiotopoulos, A. Z. J. Phys. Chem. B 2008, 112, 13783 .

(33) Maiti, P. K.; Çağin, T.; Wang, G.; Goddard, W. A. Macromolecules 2004, 37, 6236.

(34) Lin, S. T.; Maiti, P. K.; Goddard, W. A. J. Phys. Chem. B 2005, 109,8663 .

(35) Weiner, S. J.; Kollman, P. A.; Nguyen, D. T.; Case, D. A. J. Comput. Chem. 1986, 7, 230.

(36) Wang, J.; Wolf, R. M.; Caldwell, J. W.; Kollman, P. A.; Case, D. A. J. Comput. Chem. 2004, 25, 1157.

(37) Jorgensen, W. L.; Chandrasekhar, J.; Madura, J. D.; Impey, R. W.; Klein, M. L. J. Chem. Phys. 1983, 79, 926.

(38) Gasteiger, J.; Marsili, M. Tetrahedron 1980, 36, 3219.

(39) Weiner, S. J.; Kollman, P. A.; Nguyen, D. T.; Case, D. A. J. Comput. Chem. 1986, 7, 230.

(40) Posocco, P.; Ferrone, M.; Fermeglia, M.; Pricl, S. Macromolecules 2007, 40, 2257.

(41) Lee, H.; Larson, R. G. J. Phys. Chem. B 2006, 110, 18204.

(42) Cui, S. T. Phys. Rev. Lett. 2007, 98, 138101.

(43) Yao, L.; Sullivan, J.; Hower, J.; He, Y.; Jiang, S. J. Chem. Phys. 2007, 127, 195101.

(44) Hansen, C. M. J. Paint Technol. 1967, 39, 104

(45) De Brabander, C.; Van den Mooter, G.; Vervaet, C.; Remon, J. P. J. Pharm. Sci. 2002, 91, 1678.

(46) Salonen, J.; Paski, J.; Vaha-Heikkila, K.; Heikkila, T.; Bjorkqvist, M.; Lehto, V. P. Phys. Status Solidi A 2005, 202, 1629.

(47) Tanis, I.; Tragoudaras, D.; Karatasos, K.; Anastasiadis, S. H. J. Phys. Chem. B 2009, 113, 5356.

(48) Bustamante, P.; Pena, M. A.; Barra, J. Int. J. Pharm. 2000, 194, 117.

(49) Wuand, C.; McGinity, J. W. AAPS PharmSciTech. 2001, $2,24$.

(50) Vueba, M. L.; Pina, M. E.; De Carvalho, L. A. E. B. J. Pharm. Sci. 2008, 97, 845 .

(51) Twyman, L. J.; Beezer, A. E.; Esfand, R.; Hardy, M. J.; Mitchell, J. C. Tetrahedron Lett. 1999, 40, 1743.

(52) Gittins, P. J.; Twyman, L. J. Supramol. Chem. 2003, 15, 5.

(53) Santo, M.; Fox, M. A. J. Phys. Org. Chem. 1999, 12, 293.

(54) Cheng, Y.; Xu, Z.; Ma, M.; Xu, T. J. Pharm. Sci. 2008, 97, 123.

(55) Chiessi, E.; Cavalieri, F.; Paradossi, G. J. Phys. Chem. B 2007, 111,2820 .

(56) Jeffrey, G. A.; Saenger, W. Hydrogen Bonding in Biological Structures; Springer-Verlag: Berlin, 1991.

(57) Speakman, J. C. The hydrogen bond and other intermolecular forces; The Chemical Society: London, 1975.

(58) Lommerse, J. P. M.; Price, S. L.; Taylor, R. J. Comput. Chem. 1997, 18, 757.

(59) Hansen, J.-P.; McDonald, I. R. Theory of Simple Liquids, 3rd ed.; Elsevier: Amsterdam, 2006.

(60) Kanaya, T.; Kaji, K. Dynamics in the glassy state and near the glass transition of amorphous polymers as studied by neutron scattering. Polym. Phys. Eng. 2001, 154, 87.

(61) Karatasos, K.; Lyulin, A. V. J. Chem. Phys. 2006, 125, 184907. (62) Fischer, H. E.; Barnes, A. C.; Salmon, P. S. Rep. Prog. Phys. 2006, 69, 233.

(63) Jund, P.; Kob, W.; Jullien, R. Phys. Rev. B 2001, 64, 134303. 42

(64) Fujita, T.; Watanabe, H.; Tanaka, S. Chem. Phys. Lett. 2007, 434,

JP9039176 